University of New Hampshire

University of New Hampshire Scholars' Repository

10-1994

\title{
Representing Nodes and Arcs in 3D Networks
}

\author{
Glenn Franck \\ University of New Brunswick \\ Colin Ware \\ University of New Hampshire, Durham, colin.ware@unh.edu
}

Follow this and additional works at: https://scholars.unh.edu/ccom

Part of the Computer Sciences Commons, and the Oceanography and Atmospheric Sciences and Meteorology Commons

\section{Recommended Citation}

Franck, G.; Ware, C., "Representing nodes and arcs in 3D networks," in Visual Languages, 1994.

Proceedings., IEEE Symposium on , vol., no., pp.189-190, 4-7 Oct 1994 doi: 10.1109/VL.1994.363618

This Conference Proceeding is brought to you for free and open access by the Center for Coastal and Ocean Mapping at University of New Hampshire Scholars' Repository. It has been accepted for inclusion in Center for Coastal and Ocean Mapping by an authorized administrator of University of New Hampshire Scholars' Repository. For more information, please contact Scholarly.Communication@unh.edu. 


\title{
Representing Nodes and Arcs in 3D Networks
}

\author{
Glenn Franck and Colin Ware \\ Faculty of Computer Science, University of New Brunswick, P.O. Box 4400 \\ Fredericton, N.B. E3B 5A3, e-mail: glenn@UNB.ca
}

\begin{abstract}
This paper introduces six graphical principles for $3 D$ network displays. These are justified with examples from GraphVisualizer3D, a system developed by the authors to investigate the problems of $3 D$ visualization of information networks. GraphVisualizer $3 D$ enables the exploration of surface color, surface texture, object shape, arc shape and labeling conventions.
\end{abstract}

\section{Introduction}

The creators of Cone Trees claim that approximately one thousand nodes are representable using a 3D tree structure [2], considerably more than could be understood in 2D. Our recent experiments involving a 3D graph tracing task showed that test subjects were able to comprehend about three times as much information in an interactive, 3D environment as in a comparable 2D one, if both stereo viewing and motion parailax information is available [6].

When constructing a 3D diagram, the design space is enlarged considerably. Such factors as 3D form, lighting models and viewing angles become critical. This paper describes a set of design rules that we have developed to help create good representations of arbitrary networks of information in 3D space, and presents some examples we have implemented in a prototype system called GraphVisualizer3D (GV3D). The kind of graph we are interested in is one for which each node can contain an entire sub graph, and each arc can represent a bundle of arcs. This is sometimes called a compound digraph [4]. Our primary test case is the visualization of software code, and, in particular, object-oriented $\mathrm{C}++$ code for which there seems to be a natural mapping from software objects to corresponding visual objects.

\section{Semiotic Principles}

As a result of our research with the GV3D system, we have found the following six semiotic principles to be helpful in system design for 3D network representation.

Principle 1: entities should become graphical objects

Especially in 3D, a visual object takes on a concrete immediate quality that makes it both easy to remember and easy to recognize and place in the context of other objects [5]. Figure 1 illustrates some of the node representations we have developed. We chose simple boxes or cubes because we use a rectangular grid as a layout aid and because they are well suited to nesting. Families of boxes represent state information (see also principles 3,5 and 6). When a box is open it enlarges and reveals its contents. Figure 1 shows four open states: transparent, wire frame, a box with only the faces furthest from the viewer drawn (backfaces) combined with wire frame and one that combines wire frame with transparency. The advantage of the backface methods is that information behind the node of interest is hidden.

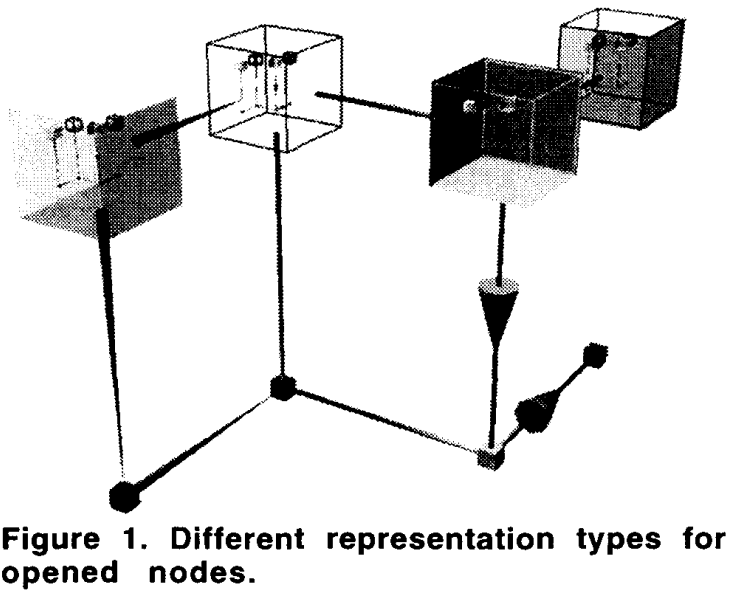

Principle 2: uses or communication relations should be represented by connecting spars Relations between the objects are naturally represented by connecting lines or arrows in a graphical representation. When these relationships are portrayed in $3 \mathrm{D}$ the lines become solid spars that run between two nodes. We have implemented six different arc representation, consisting of both solid spars and lines. We have tried a number of graphical devices to indicate the direction of a directed arc and some of these are illustrated in Figure 1. The simplest direction indicator is a color change along the length of the arc and this can give a reliable sense of direction [1]. Other, more complex representations include eight sided pyramids and 3D arrows placed in a connecting line. We have found these to be useful in different circumstances; for example, for inheritance relations we use the fat pyramidal arc while for uses relations we use the arrow. 
Principle 3: attribute relations should be represented by surface and shape properties Color and texture are both excellent in distinguishing between different node types because in nature they distinguish between related classes of objects. Shape is also appropriate (see Figure 2).

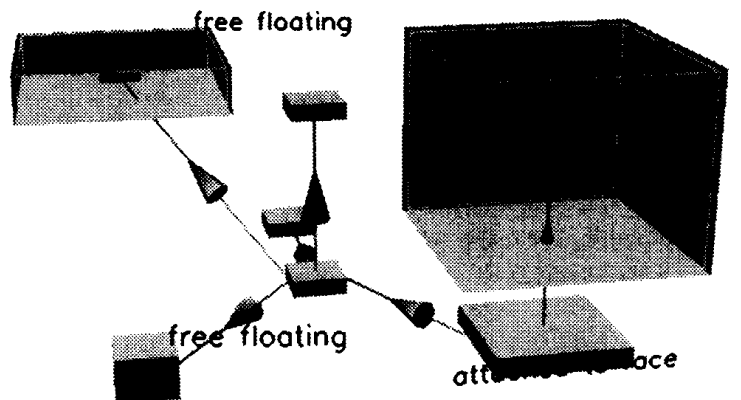

Figure 2. A portion of a network showing nodes of various sizes in both open and closed states, with two labeling methods.

Principle 4: attribute representations should be orthogonal

As far as possible, we must design orthogonal families of attribute representations. An example of a non-orthogonal set would be a box that could be rendered either filled or in wire frame. Clearly in wire frame mode the color appearance will not as easily be seen. The theory of integral versus separable display dimensions applies here [3]. In general it would be better to use separable display dimensions such as orientation and color because these can be perceived independently. In order to facilitate different yet recognizably similar objects we generate families of graphical dimensions.

- Families by form

By varying the shapes and sizes of objects, we can convey the impression of different types and amounts of information. The state of a box must be represented by changes in form that preserve identity.

- Families by color and transparency

Color has three dimensions and we may choose to render different objects of the same family with, for example, the same hue (for identity) but different saturations (for state). Hue may be very good for labeling attributes in this way because hue changes are perceptually associated with the properties of the materials out of which an object is made. In a rendered $3 \mathrm{D}$ environment it is important that the surface color be orthogonal to the effects of shading. This makes surface lightness a poor choice for displaying attributes. Transparency allows the preservation of identity while showing open and closed states.

- Families by texture

Visual textures can be arranged in order by orientation or size [7]. A problem with texture is that the appearance of most textures changes with scale, unless it is fractal in nature.
Principle 5: preservation of object constancy Objects must maintain their identity despite scale changes, viewing from different angles and distance and state changes. The following graphical devices help.

- Symmetry about a vertical axis helps make an object recognizable from different directions.

- Animated state changes preserve identity.

- Rotating labels so that the textual labels always face directly on to the user. (illustrated in Figure 2.)

Principle 6: recursive implementation of design rules

If nesting is to be defined recursively, then the representation scheme must be graphically recursive. In GV3D, we instantiate this principle by allowing each node to contain an entire graph, wherein each node of that graph can contain an entire graph, and so on. In Figure 1, for example, the entire high-level graph of eight nodes and arcs is repeated inside each open node.

\section{Conclusions}

We feel that representing networks of nodes and arcs in 3D has clear benefits in that it enlarges the perceptual space available for information display. However, the costs of a transition to $3 \mathrm{D}$ data representations are considerable. A new set of diagrammatic conventions must be developed that map information structures to three dimensional objects in the display space; this is the problem of 3D semiotics and is the issue we have begun to address here.

\section{Acknowledgments}

We are indebted to Bruce Simpson of the University of Waterloo for the idea to use spars to represent arcs. NSERC provided funding. Arthur Ryman of IBM Toronto Labs and Tim Dudley of Bell Northern Research provided support and encouragement.

\section{References}

1. Fowler, D. and Ware, C. Strokes for Representing Univariate Vector Field Maps, Graphics Interface Proceedings (1989), pp. 249-253.

2. Robertson, G.G., Mackinlay, J.D and Card, S. K., Cone Trees: Animated 3D Visualizations of Hierarchical Information. CHI'91 Proceedings. (1991) pp.189-194.

3. Smith, L, and Evans, P. Similarity, Identity and Dimensions: Perceptual Classification in Children and Adults, in Object Perception: Structure and Process, Shepp and Ballesteros, Eds, Lawrence Erlbaum 1989, 325-357.

4. Sugiyama, K., and Misue, K., Visualization of Structural Information: Automatic Drawing of Compound Digraphs, IEEE Transactions on Systems, Man and Cybernetics, Vol. 21, No. 4, July/August 1991, pp. 876-892.

5. Ware, C., The Foundations of Experimental Semiotics, Journal of Visual Languages and Computing, 4 (1993), pp. 91-100.

6. Ware, C. and Franck, G., Visualizing Information Nets in Three Dimensions, University of New Brunswick technical report TR94-082, February, 1994.

7. Ware, C. and Knight, W., Using Visual Texture for Information Display, ACM Transactions on Graphics (in press). 